\title{
A Clock for the \\ Manufacturing Systems Integration Testbed
}

\section{Don Libes}

\author{
U.S. DEPARTMENT OF COMMERCE \\ National Institute of Standards \\ and Technoloty \\ Manufacturing Engineering Laboratory \\ Gaithersburt, MD 20899
}





\section{A Clock for the \\ Manufacturing Systems \\ Integration Testbed}

Don Libes

U.S. DEPARTMENT OF COMMERCE National Institute of Standards and Technology

Manufacturing Endineoring Laboratory Gaithersbure, MD 20899

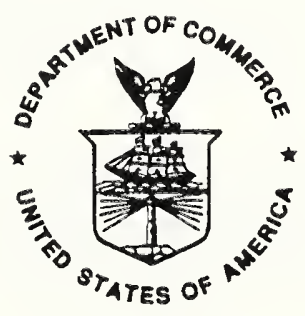

US. DEPARTMENT OF COMMERCE Robert A. Mosbacher, Secretary NATIONAL INSTIUUTE OF STANDARDS NND TECHNOLOGY

John W. Lyons, Director 



\title{
A Clock for the Manufacturing Systems Integration Testbed
}

\author{
Don Libes \\ National Institute of Standards and Technology \\ Gaithersburg, MD 20899 \\ libes@cme.nist.gov
}

\begin{abstract}
This paper describes a software module that provides timing services to the MSI, a Manufacturing Systems Integration Testbed in the automated factory.

The software "alarm clock" provides services to other MSI software including:

- synchrony,

- real-time, or non-real-time adjusted in a variety of ways,

- alarms at relative or absolute internals.

By providing a central time service, these services are provided more reliably, efficiently, and flexibly than could any client on its own.

This paper describes the implementation, interfaces, and how to design and write programs that use it.
\end{abstract}

Keywords: manufacturing, MSI, CIM, time, timing, clock, UNIX.

This work is partially supported by the Navy Manufacturing Technology Program and was prepared by U.S. Government employees as part of their official duties and is therefore a work of the U.S. Government and not subject to copyright.

Trade names and company products are mentioned in the text in order to adequately specify experimental procedures and equipment used. In no case does such identification imply recommendation or endorsement by the National Institute of Standards and Technology, nor does it imply that the products are necessarily the best available for the purpose. 


\section{Background}

The Manufacturing Systems Integration (MSI) project [Senehil, Senehi2] is developing an integrated production planning and control environment. Its aim is to provide a testbed for production management architectures integrating process planning, production planning and shop floor control.

The current MSI implementation consists of a large number of software processes running simultaneously and communicating with each other. The MSI alarm clock provides time-related services for the MSI. All communication with the clock occurs using the NIST Network Common Memory [Libes].

\section{Introduction}

An important function of the MSI is scheduling. For example, resources (e.g. machines) are scheduled in advance (when possible) to optimize throughput. In order for schedules to be useful, their times must be interpreted consistently by other MSI processes. This synchronization is provided by the clock which is the only source of time in the testbed. (We will use "time" to imply "time and date" unless otherwise specified.) The implementation provides this very efficiently, essentially by automatically providing a time stamp with every network input.

The clock keeps "MSI time". MSI time can be made to track "real time". More typically, the clock can be reset to a particular time. This is very useful since MSI schedulers currently produce absolute times. Rather than reproducing or editing schedues, the clock can be adjusted backwards to rerun an old schedule. The clock can also scale time. For example, the clock can tick once per second for every real hour (for watching fast operations in detail), or vice versa (for watching macro behavior). The clock can run at virtually any rate, including backwards (although we have not found a use for this last one, yet).

The clock can deliver "alarms". Analogous to a bedside alarm clock, MSI clients may request alarms which "wake" themselves up at times of interest. For instance, according to a schedule it may be possible to determine that a process has nothing to do for the next 15 minutes. In this case, it is desirable to "sleep" until then, allowing a CPU more efficiency in serving other processes. The implementation provides optimum efficiency by waking a process when either an alarm occurs or input has arrived (the only other reason to wake up). The clock can keep track of an unlimited number of alarms.

The clock provides a variety of other features such as stopping time, single-stepping, and advancing directly to the next alarm. In addition, the clock understands time in several high-level formats.

\section{Status}

Prior implementations were inaccurate and inefficient. Processes were forced to busy-wait or estimate future clock movement. There was no alarm mechanism. Processes were also required to do date conversion and comparisons themselves. There was no means of stepping or changing the movement or rate of time. 
The current clock (described in this document) keeps time accurately and efficiently. The "advance time to the next alarm" is an especially appreciated feature. The clock was successfully used in the October ' 90 AMRF test run.

Several enhancements would be useful. They are:

- Publishing extant alarms in common memory. This would be helpful in debugging and gaining a feel for the status of the clock clients.

- An earlier implementation of the clock used non-portable interfaces. The current clock continues using these for compatibility. These should be deprecated.

\section{Starting the clock}

The clock is implemented as a UNIX program. Assuming your path is correct, the clock is invoked by the command:

$$
\text { alarm_clock }
$$

The clock has several optional arguments. These can appear in any order:

$-\mathrm{cm}\langle$ host>

The host from which to get common memory service. The default host is the current host.

$\mathrm{mm} / \mathrm{dd} / \mathrm{yy}$

Numeric values of the month, day, and year to initialize the clock. The default values are the current date. The year is assumed to be biased by 1900, so that only the last two digits should be specified. Numbers do not require leading zeros.

hh $: m m: s s$

Values of the hours, minutes, and seconds to initialize the clock. The default values are the current time. Numbers do not require leading zeros.

+debug

Enables debugging output. This includes each time a new time/date or alarm occurs, or when a new command is received. By default, debugging is disabled (-debug).

-warn

Enables warning output. This includes such things as invalid commands or alarm requests. By default, warning is enabled (+warn).

\section{Clock user interface}

The clock accepts commands through a common memory interface. Normally, this is done by the gti program [Sauder]. This section provides additional information to supplement Sauder's documentation, but does not attempt to duplicate it. Please refer to it if there are further questions.

Clock commands are as follows:

Set Time

Hours, minutes, and seconds of MSI time are set to those given in the command. 
Set Date

Month, day, and year of MSI time are set to those given in the command.

Set Realtime

MSI time is set to EST. Note that the rate is not changed.

Zero Time

Hours, minutes and seconds of MSI time are set to 0.

Set Rate

The rate is set. MSI time moves rate times faster than real time. The default rate is 1 . (See Step Time.)

Stop Time

Time is stopped until the next Start Time command.

Start Time

Time is started if not already running.

Step Time

The time is advanced to that of the next alarm. If no alarms exist, time is incremented by rate seconds.

Kill Clock

The clock process exits. It will accept no commands after this.

Status Report

No action (other than default).

Note that the gti program does not give entirely clean access to the clock. For example, while changing the time, gti also stops the clock. Inexplicibly, this does not occur while changing the date.

The commands are available to the C programmer as the enumerated type, clock_command, which is defined in the file, msi_clock_command.h. This file also defines two common memory variables via the preprocessor macros: MSI_CLOCK_CMD and MSI_CLOCK_STS.

Commands are written to the variable defined by MSI_CLOCK_CMD in a non-portable format described by the $\mathrm{C}$ structure:

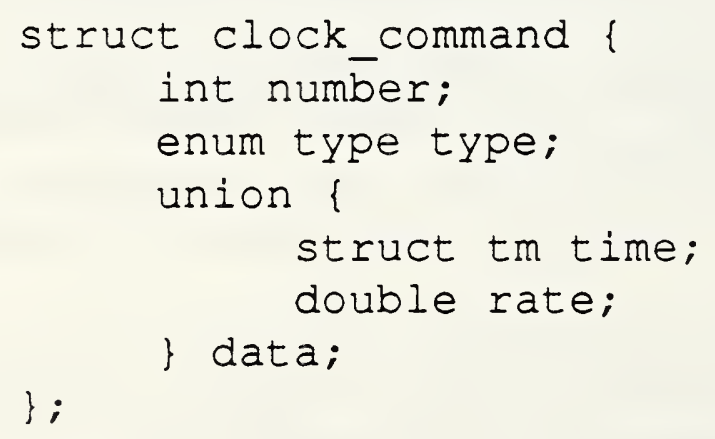

In response to each command, the clock writes a status reported to the variable defined by MSI_CLOCK_STS in a non-portable format described by the C structure:

struct clock_status f 


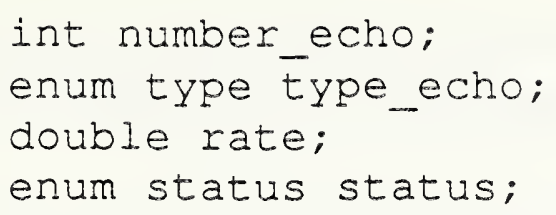

The command element number is transferred to status element number_echo. Similarly, so is type. type is also interpreted as a clock command as described above.

Depending on the type, appropriate elements are extracted from the union data. The current rate is always echoed in the clock status.

Finally, the status element is set to one of running, stopped, or killed as appropriate. The actual values of these and other enums may be found in the source code.

The clock status interface allows no way of returning command errors. Errors are printed on stdout (if +warn is in effect).

\section{Clock program interface}

The clock provides two services to MSI client programs. Namely, time service and alarm service. In order to reduce application programming as much as possible, a client-side $C$ library exists which encapsulates the implementation of MSI clock services, leaving actual usage as the simplest of programming tasks. Programmers that cannot use the client-side C library (i.e., Symbolics users) should refer to "Implementation Notes" (below).

An example of most of the services described below exists in $\sim \mathrm{msi} / \mathrm{src} /$ alarm_clock/test_service.

In order to use the clock service library, the following steps are required:

1) You must include a header file defining the library interface. In particular, you should add the following line to the beginning of any $\mathrm{C}$ source file that has calls to the clock services.

$$
\text { \#include "msi_clock_service.h" }
$$

2) You must call msi_clock_init before calling any other clock services. msi_clock_init returns a 0 normally, or a -1 upon failure. For example:

$$
\text { if }(-1==\text { msi_clock_init ()) exit }(-1) \text {; }
$$

Since the clock uses common memory, you should also call $\mathrm{cm}$ init, if you do not do so elsewhere. cm_init should be called before msi_clock_init.

3) Any files that make clock service (or common memory, for that matter) calls should be compiled with additional - I flags to tell the $\mathrm{C}$ compiler where it can find the include files. For example:

$$
\begin{aligned}
& \text { cc-I/home/lurch/msi/src/alarm_clock -I/usr/local/include/cm } \\
& \text {-c prog.c }
\end{aligned}
$$


4) Object files must be linked with the appropriate libraries. For example:

$$
\begin{aligned}
& \text { cc prog.o /home/lurch/msi/src/alarm_clock/msi_clock_servi- } \\
& \text { ce.o-lcm -lstream }
\end{aligned}
$$

5) The common memory manager and the alarm clock must be running before you run your program(s). The common memory manager must be run first. For example:

$$
\begin{aligned}
& \mathrm{cmm} \& \\
& \text { alarm_clock \& }
\end{aligned}
$$

\section{Time}

When the clock is running (or if it is being stepped or set by hand), it writes the latest MSI time to the common memory. (The actual rate at which time is written is once per MSI second up to MAX_RATE times per real second. MAX_RATE is a compile-time constant currently equal to 3.)

Each time your local common memory is updated by other common memory variables (or by explicit request), you receive the latest copy of the MSI time. (Time is not sent on its own, because it is written too frequently for most processes to keep up.)

Time is transmitted as a 4-byte integer (most significant byte first) representing seconds since January 1,1970 . It is stored locally as a time_t. time_t can be directly manipulated by the usual $\mathrm{C}$ arithmetic operators, and printed out with printf using the \% $\mathrm{d}$ format.

UNIX provides a number of library routines for high-level manipulation of a time_t including ctime(3) which converts it to an ASCII representation, and localtime(3) which breaks it in to a form in which individual elements (hours, minutes, etc.) are directly manipulable.

This representation of time may be retrieved with the following function.

$$
\text { time_t msi_time_seconds(char *time) }
$$

The function msi_time_seconds returns the number of seconds equivalent to the time described by its argument. Its argument may be 0 , in which case, the current MSI time is returned. Alternatively, the "standard MSI ASCII time representation" may be used.

The standard MSI ASCII time representation is of the form "yyyymmddhhmmss". All fields must be zero-filled. For example, "19901231190123" represents December 31, 19:01:23 1990. This form may be directly produced by the following function:

$$
\text { char *msi_time_printable(time_t time) }
$$

Given a number of seconds since January 1, 1970, the function msi_time_printable returns the standard MSI ASCII time representation. If time is 0 , the current MSI time is used. This function returns a pointer to a static buffer which will be reused when this function is next called. 
The following C program prints the current MSI time in both formats. Error checking has been omitted for readability.

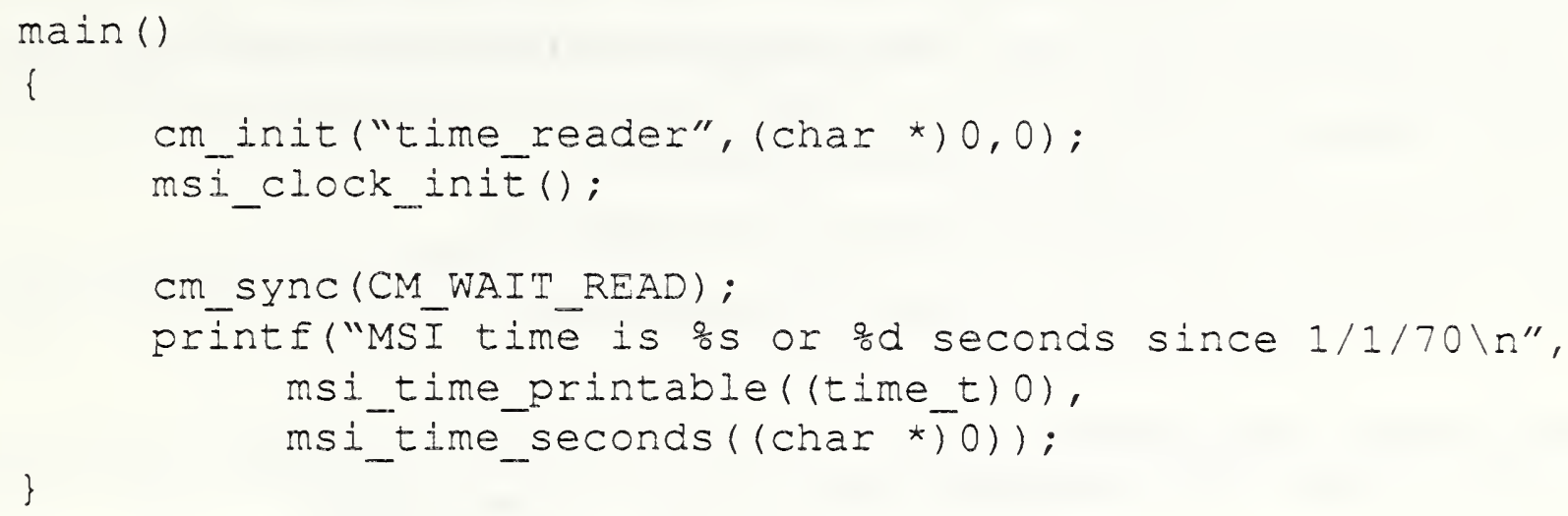

Note that CM_WAIT_READ (meaning "force the common memory server to synchronously update all my variables") is an expensive $\mathbf{c m}$ _sync option which is invariably not required by real programs, but is necessary in this example.

As mentioned earlier, $\mathrm{C}$ arithmetic operators can work directly on time_t values. This is not the case for MSI printable values. The following macros are provided as a convenience for comparing two MSI printable values. They are actually more efficient than converting both values via msi_time_seconds.
int msi_time_eq $(x, y)$ returns 1 if $x==y, 0$ otherwise.
int msi_time_lt $(x, y)$ returns 1 if $x<y, 0$ otherwise.
int msi_time_gt $(x, y)$ returns 1 if $x>y, 0$ otherwise.
int msi_time_ge $(x, y)$ returns 1 if $x>=y, 0$ otherwise.
int msi_time_le $(x, y)$ returns 1 if $x<=y, 0$ otherwise.
int msi_time ne $(x, y)$ returns 1 if $x !=y, 0$ otherwise.

\section{Alarm}

The clock will write to a named common memory variable at a requested time, in effect, providing an alarm service. Alarms are manipulated by calling the following functions:

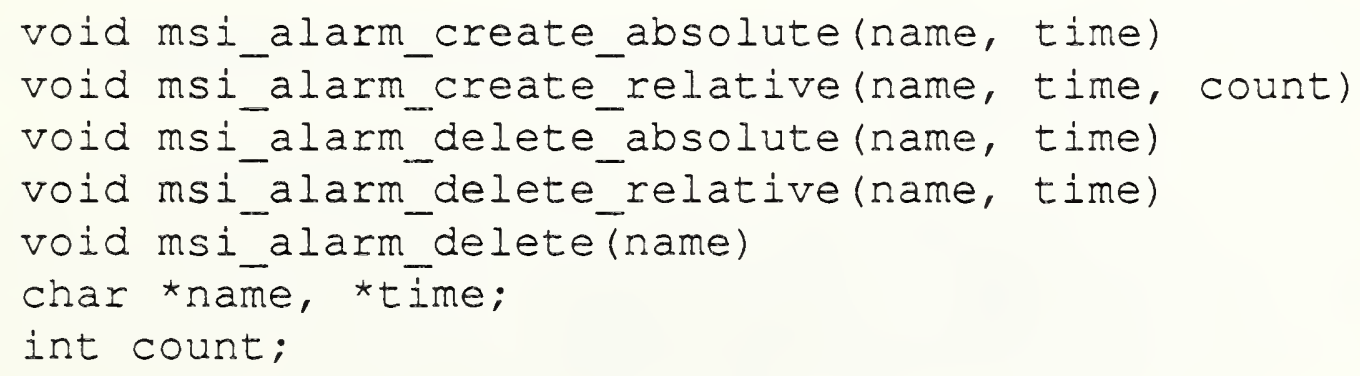

In all of these calls, name indicates the common memory variable to be written at the given time. This variable must be declared by the user as follows: 


$$
\text { cm_declare ("var", CM_ROLE_READER/CM_ROLE_WAKEUP) ; }
$$

The time may be specified either using the standard MSI ASCII form (described) above, or using one or both of the strings "mm/dd/Yy" and "hh:mm: ss" (separated by whitespace) in either order. The latter style does not require leading zeros.

The formats will be repeated and discussed individually here.

$$
\text { void msi_alarm_create_absolute (name, time) }
$$

msi_alarm_create_absolute creates an alarm for an absolute time/date. After occurring, the alarm is deleted. (This is important, since time can be set backwards.)

$$
\text { void msi_alarm_create_relative(name, time, count) }
$$

msi_alarm_create_relative creates an alarm for a relative time. The alarm will occur count times in the future, each spaced the indicated date/time from one another. A zero count indicates infinity. If the clock is advanced, alarms scheduled for a prior time will immediately occur, respacing from that point. If the clock is set back, relative alarms will not begin triggering until the previous (now future) time is reached.

Note that months and days are essentially meaningless in relative date specifications and are therefore ignored. However, for readability, they must be specified (as zeros).

For example, the following program sets an alarm for 10 seconds in future, and then wakes at that time.

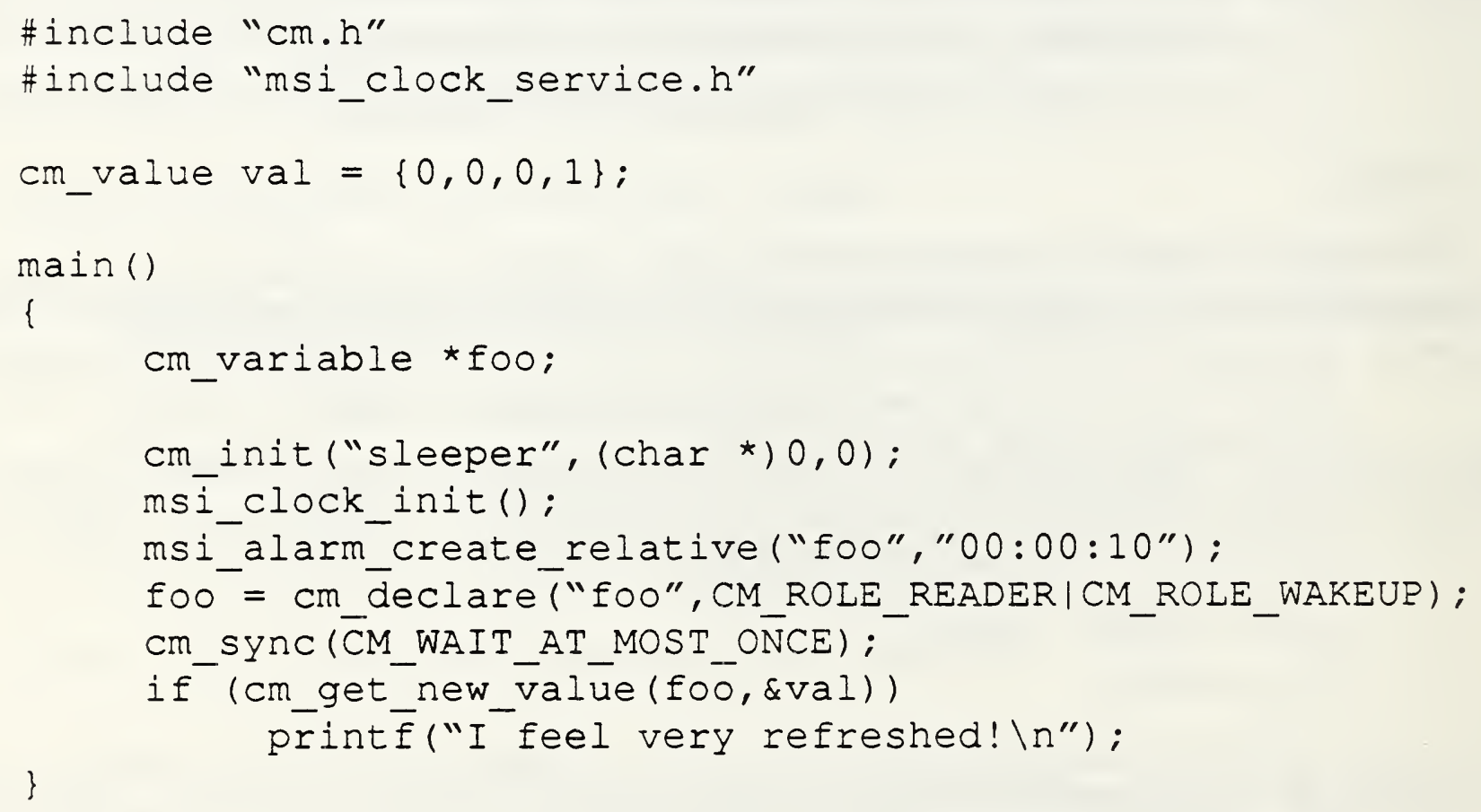


void msi_alarm_delete_absolute (name, time)

msi_alarm_delete_absolute removes an absolute alarm set for the given time. If no time is specified, all absolute alarms which match the name are deleted.

void msi_alarm_delete_relative(name, time)

msi_alarm_delete_relative removes a relative alarm set for the given time. If no time is specified, all absolute alarms which match the name are deleted.

void msi_alarm_delete (name)

msi_alarm_delete removes all alarms associated with the given name.

The clock will remember an unlimited number of alarms. Multiple alarms may be associated with the same variable.

Alarm variables should be named in a way that distinguishes them from one another and from other variables. For example, append the process name with the string "_alarm", such as in "hws_alarm". Of course, a process may have multiple alarm variables if $\bar{d}$ esired.

The actual implementation of these functions has ramifications on their use. In particular, cm_sync must be called after each of these functions in order to get them to work. More importantly, since they use the same common memory variable internally to communicate with the alarm clock, if you do not call cm_sync between alarm clock calls, the former of the two alarm requests will be overwritten by the latter request. The implementation avoids calling $\mathbf{c m}$ _s snc (for you) because doing so can prevent use of common memory's reliable queuing mechanism.

Since common memory clients have no way of knowing if anyone is reading a common memory variable, the clock has no way of telling if a client has died. Thus, the clock will deliver an alarm that a client has requested, even if the client has long since exited. If there is a chance that client software may be restarted with alarms pending, I recommend you initialize your system by deleting any alarms for which you are responsible. This will prevent alarms from a previous incarnation triggering mysteriously (to you).

\section{Implementation notes}

The following implementation notes are probably not of interest to the casual user of the MSI clock. They describe precise details of the common memory interface.

\section{Common memory time interface}

The time interface is implemented by reading from the variable specified by the macro MSI_TIME. This should be declared CM_ROLE_READER. It is almost certainly wrong to declare it as CM_ROIE_WAKEUP, as the clock can easily overrun your input buffers this way.

As described above, time is transmitted as a time_t value most significant byte first. After your local common memory has been updated, you can read it by calling $\mathbf{c m}$ _get_value and then converting it to native form (e.g., ntohl on a Sun). The following program prints the current date. 


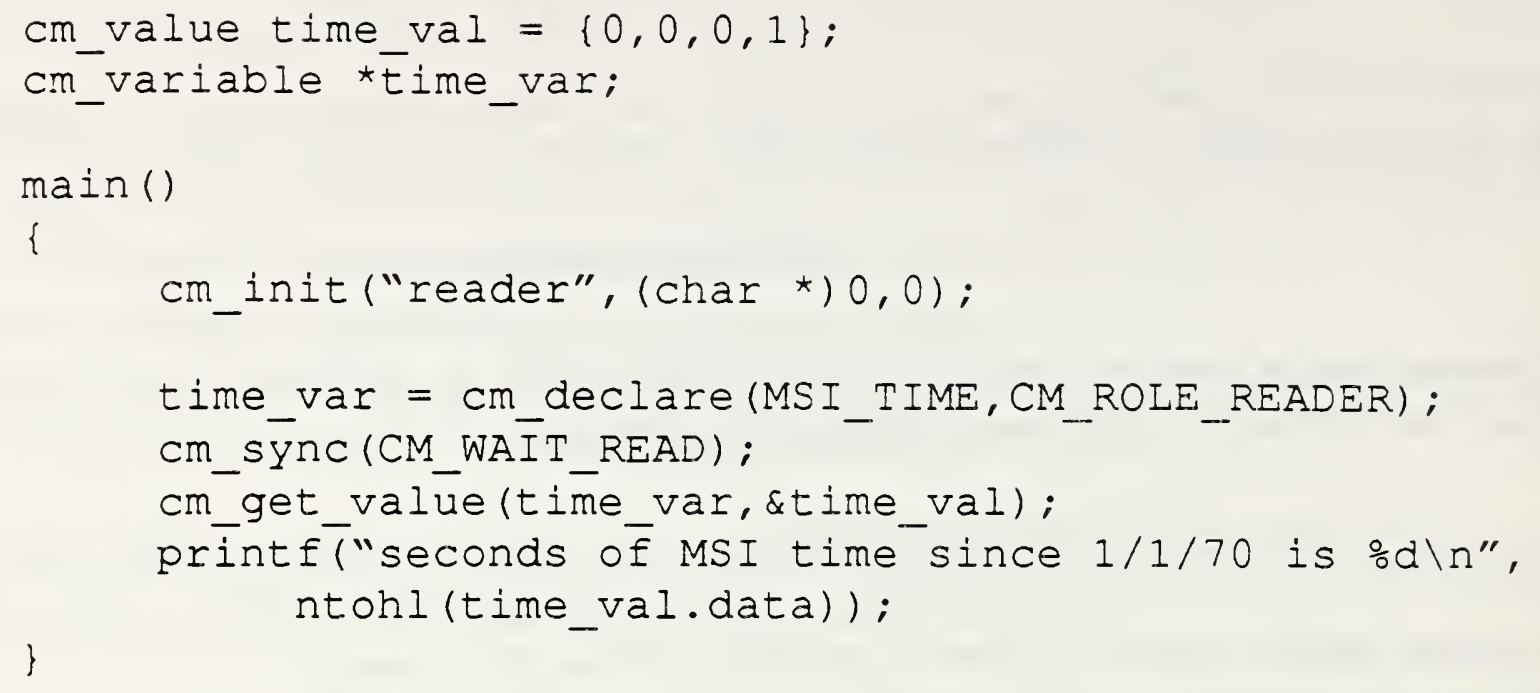

\section{Common memory alarm interface}

The alarm interface is implemented by writing requests to the variable specified by the macro MSI_ALARM_REQUEST. This is a shared variable and should be declared CM_ROLE_NONXWRITE.

Requests are ASCII strings in the following format:

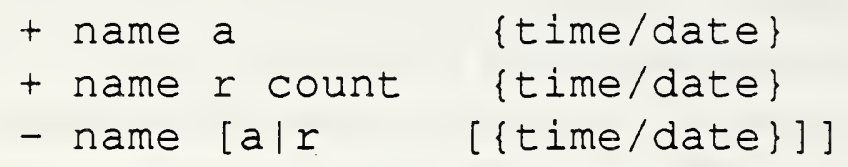

The initial + or - indicates whether an alarm is created or deleted. The name indicates the common memory variable to be written at the given time/date. The a or $\mathrm{r}$ describe whether the time/ date are to be used absolutely or relatively as described earlier. The following example, sets an alarm for 10 seconds in the future, and then wakes up at that time.

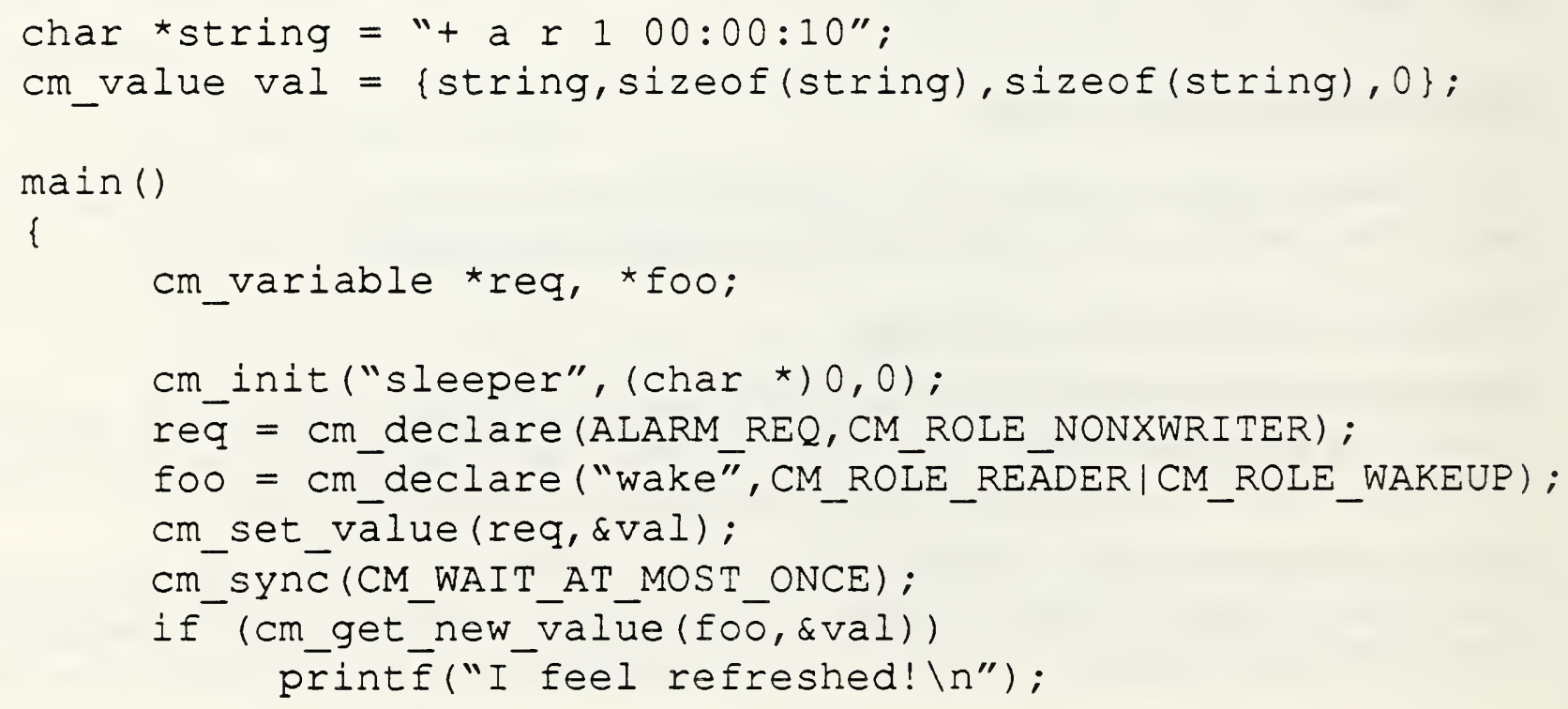




\section{Status reporting}

The clock interfaces provide no way to deliver error messages from the clock to a client or userinterface. This should be remedied. In the meantime, warnings are printed to the clock's stdout, and errors to the clock's stderr.

Warnings and errors are self-explanatory.

\section{Testing}

The program sleeper (in $\sim$ msi/src/alarm_clock) is a test program that will report any alarm activity on the common memory variables $\mathbf{a}, \mathbf{b}, \mathbf{c}, \mathbf{d}, \mathbf{e}$, and $\mathbf{f}$ along with the MSI time at each alarm. This is particularly useful for testing the alarm.

A separate program, such as realuser, must be used to write alarm requests.

\section{Portability}

Due to the original design of the command/status interfaces, the clock itself will not be able to communicate with user interface programs if they or the clock itself runs on a different hardware/ software base than is currently being used, a MC680x0-based computer running SunOS.

The command/status formats used by the user interfaces currently use native integer and floating point storage formats, which differ from computer to computer. This should be corrected.

The interfaces used for time and alarm request and delivery are portable to any POSIX-conforming system.

\section{Source Code}

The source code for the clock can be found in $\sim \mathrm{msi} / \mathrm{src} /$ alarm_clock. This code is extremely subtle and should not be modified without careful thought. The code is very carefully written to generate accurate time and alarms, despite servicing alarms and other requests.

The basic loop is shown below.

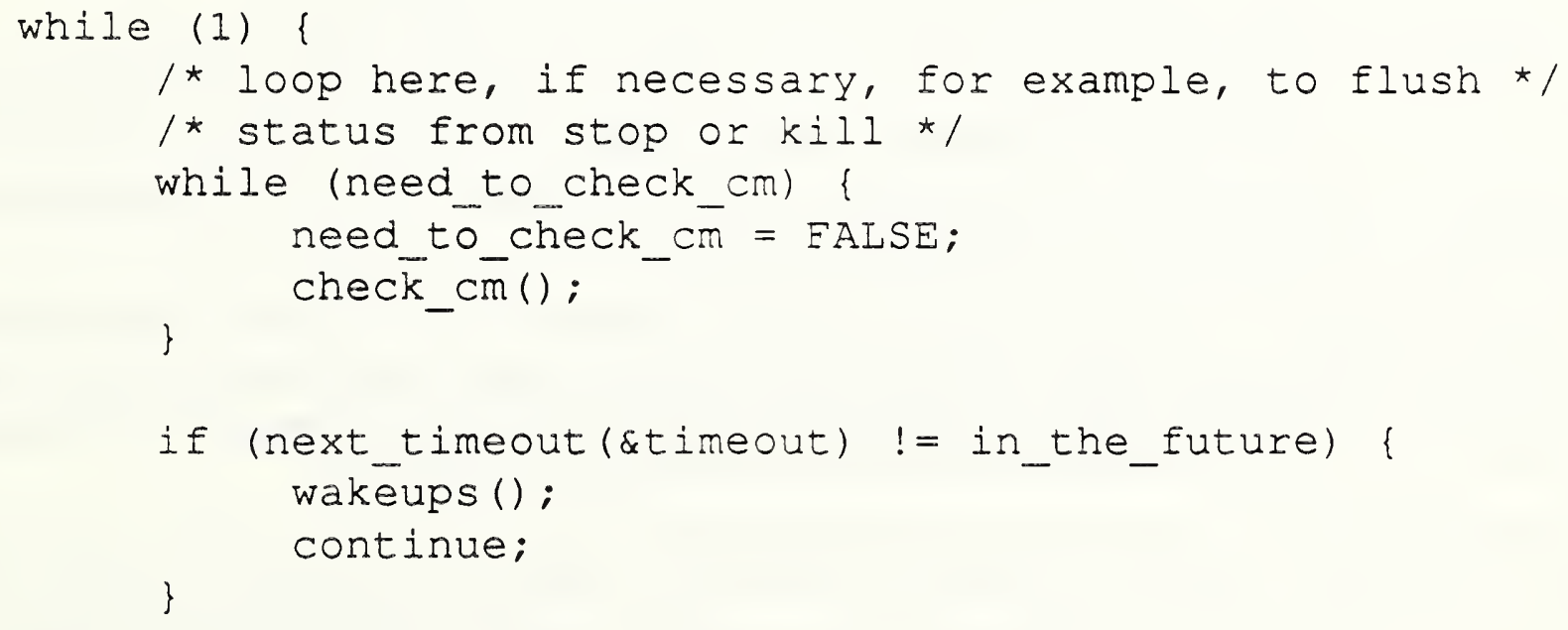




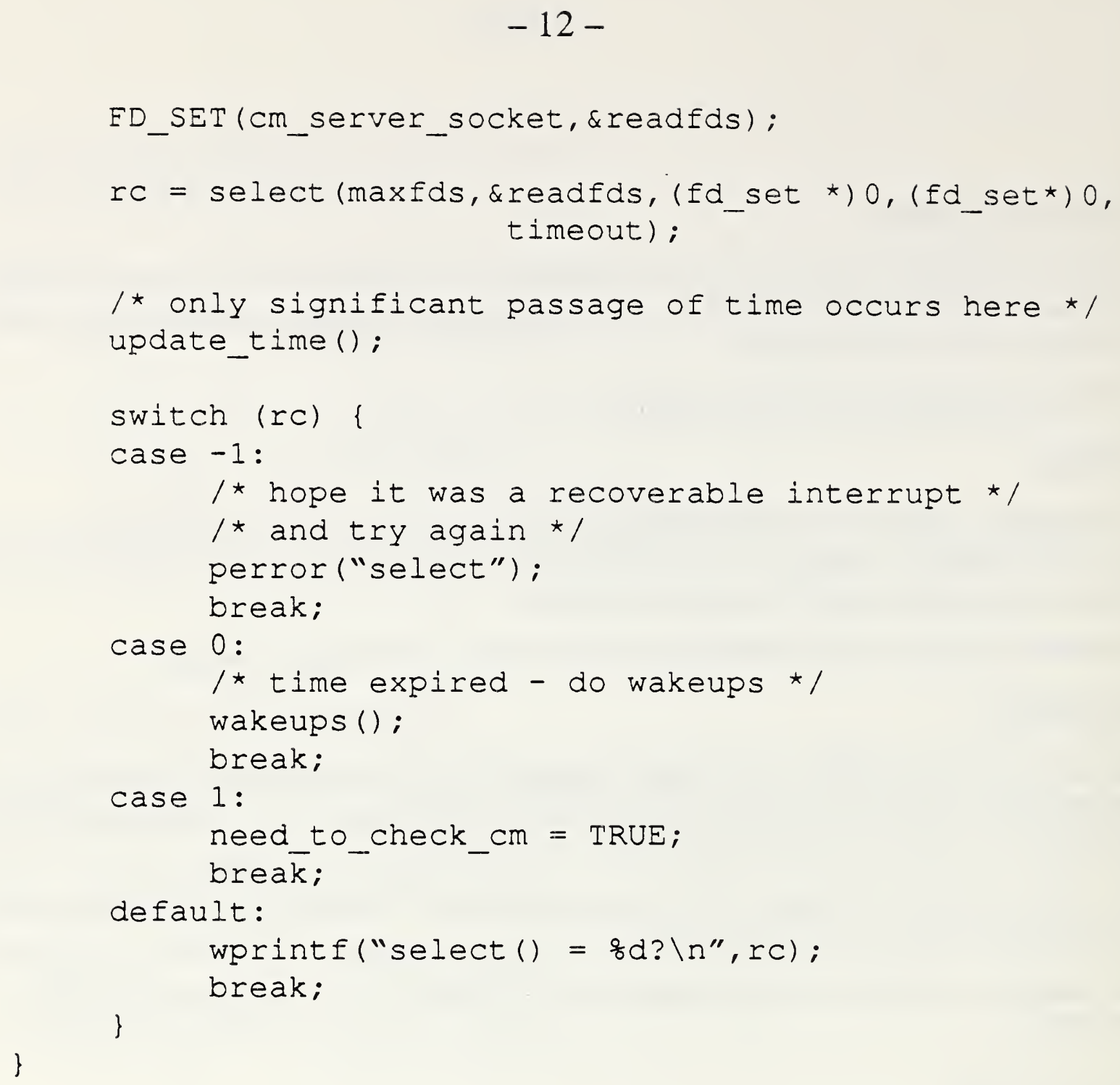

The first important function is next_timeout which decides what passage of time to wait for. This function examines pending alarms, as well as the current clock rate to decide if the clock, itself, can sleep and if so for how long. Even if the clock isn't running, an alarm might still have to be generated, if, for example, the time was advanced by an explicit command. If any alarms are ready to be delivered, wakeups delivers them on the following statement.

Note that this is immediately followed by a $C$ continue statement which drives control to the top of the loop. At the top is a check for common memory. If any alarms need to be delivered, common memory will need to be flushed. (need_to_check_cm is set when any common memory variable has been written.) On the other hand, we do not want to sync with common memory if unnecessary since this is a (relatively) slow operation.

Other fast operations such as requests to stop or set the clock will be picked up and executed when common memory is checked at this point. Rather than putting logic inside of check_cm to loop internally, it is much simpler to recall it immediately after it has completed. This may seem strange, but it is much cleaner and simpler than writing it any other way.

Once any alarms are flushed, the alarm clock sleeps in such a way that it will be woken up only if the (real) time it wanted to awaken has arrived, or a service requested has arrived via common memory. Because of our avoidance of unnecessary common memory synchronization, we can 
consider that the only lengthy passages of time occur while the clock is asleep-i.e., during select. Thus, this is immediately following by a call to update_time which updates the clock's idea of the time.

update_time also publishes the new time in common memory. (Actually, it only writes a common memory variable and preps need_to_check_cm, so that it will be written out to common memory when the top of the loop is reache $\bar{d}$, which will be immediately after this.)

update_time (below) looks trivial but is very subtle. msi_time is (or shortly will be) the current msi_time. old_msi_time is not the previous value of msi_time, but is the time of the last MSI time that was last specficially requested by command. old_tv_real_time is the corresponding real time when old_msi_time was set. Similarly, tv_real_time is the current real time. By subtracting these, scaling by the rate, and adding the result to the old_msi_time, the new MSI time is determined.

The key is not to use the MSI time computed during the previous iteration. The problem is that miniscule fractions of time creep in between when the time is sampled and the new value is computed. By recomputing the new time from a known-to-be-correct baseline each time, we avoid any time slop that might build up.

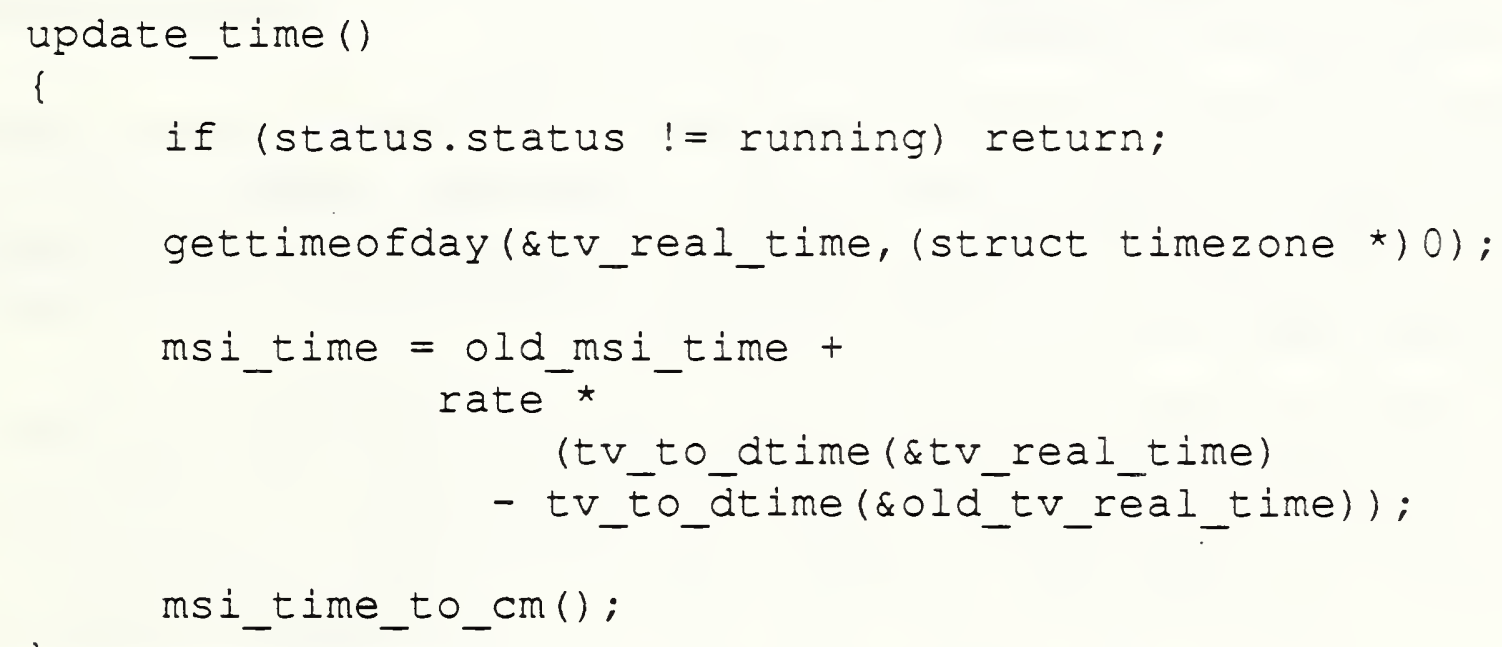

Following update_time, we determine why the clock stopped sleeping. This can be one of four cases.

-1 or default means an error occurred. We have never actually seen this happen.

0 means no service request occurred, and thus the clock slept for the time it intended to. Since this could very well been for an alarm, wakeups is called to find out. wakeups will actually find all alarms (several may be set for the same time) that have the current or a previous time.

1 means that a service request occurred via common memory. Control is passed back to the top of the loop so that common memory can be synchronized and the request received. 



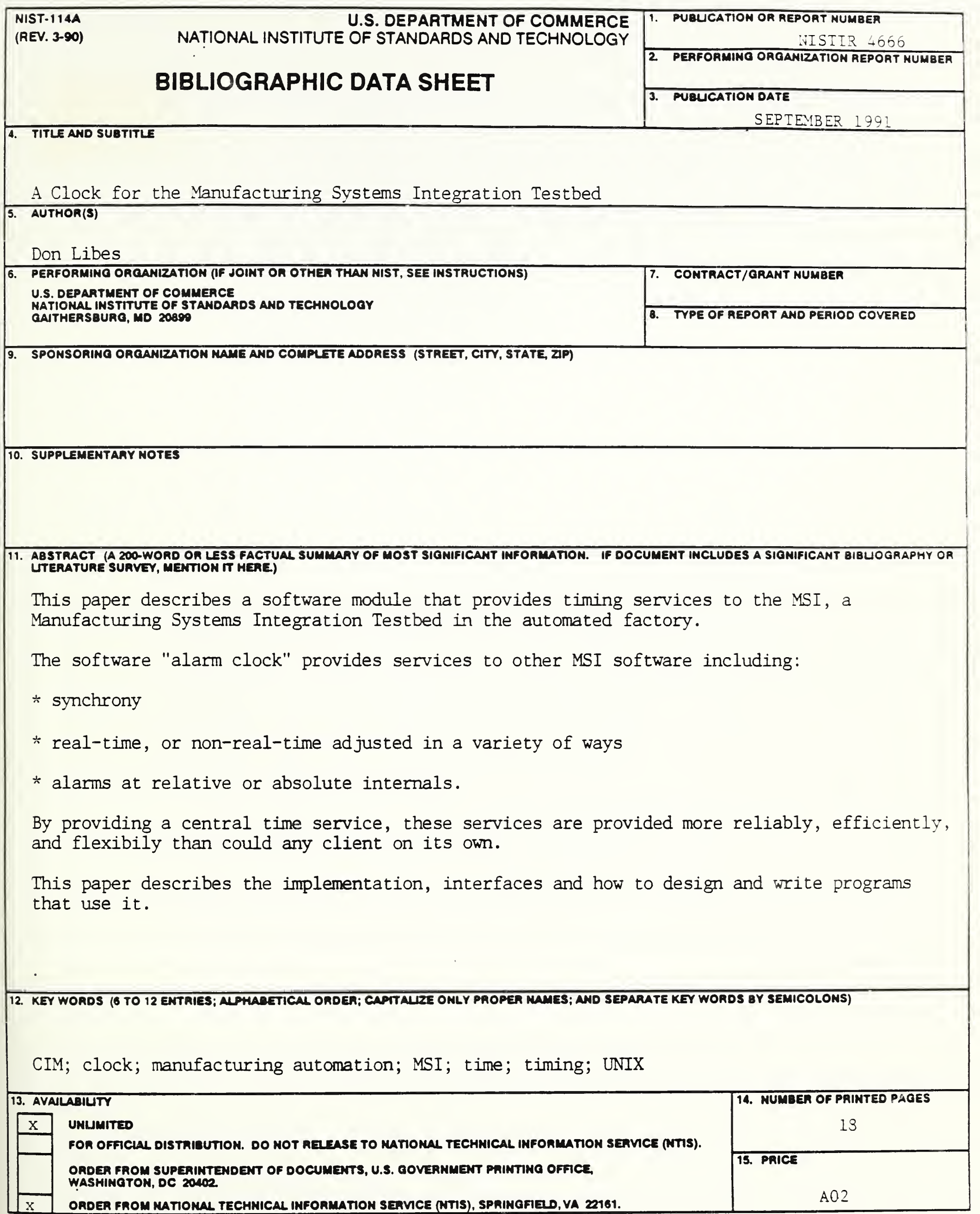




